\title{
OPTIMAL ASYMPTOTIC BOUNDS FOR DESIGNS ON MANIFOLDS
}

\author{
BIANCA GARIBOLDI AND GIACOMO GIGANTE
}

\begin{abstract}
We extend to the case of a $d$-dimensional compact connected oriented Riemannian manifold $\mathcal{M}$ the theorem of A. Bondarenko, D. Radchenko and M. Viazovska 2 on the existence of $L$-designs consisting of $N$ nodes, for any $N \geq C_{\mathcal{M}} L^{d}$. For this, we need to prove a version of the MarcinkiewiczZygmund inequality for the gradient of diffusion polynomials.
\end{abstract}

\section{INTRODUCTION}

Let $\mathcal{M}$ be a connected compact orientable $d$-dimensional Riemannian manifold without boundary with normalized Riemannian measure $d \mu$, such that $\mu(\mathcal{M})=1$. We shall denote the Riemannian distance between $x$ and $y$ by $|x-y|$. Let $\left\{\varphi_{k}\right\}_{k=0}^{+\infty}$ be the eigenfunctions of the (positive) Laplace-Beltrami operator, with eigenvalues $0=\lambda_{0}^{2}<\lambda_{1}^{2} \leq \lambda_{2}^{2} \leq \ldots, \Delta \varphi_{k}=\lambda_{k}^{2} \varphi_{k}$.

The space of diffusion polynomials of bandwith $L \geq 0$ is

$$
\Pi_{L}=\operatorname{span}\left\{\varphi_{k}: \lambda_{k} \leq L\right\} .
$$

We say that a set of points $\left\{x_{j}\right\}_{j=1}^{N} \subset \mathcal{M}$ is an $L$-design if

$$
\int_{\mathcal{M}} P(x) d \mu(x)=\sum_{j=1}^{N} \frac{1}{N} P\left(x_{j}\right), \quad \text { for all } P \in \Pi_{L} .
$$

Observe that since the above identity is trivially satisfied by constant functions, and since by orthogonality of the eigenfunctions $\varphi_{k}$,

$$
\int_{M} \varphi_{k}(x) d \mu(x)=0, \quad \text { for all } k \geq 1,
$$

then $\left\{x_{j}\right\}_{j=1}^{N} \subset \mathcal{M}$ is an $L$-design if and only if

$$
\sum_{j=1}^{N} \frac{1}{N} P\left(x_{j}\right)=0, \quad \text { for all } P \in \Pi_{L}^{0},
$$

where $\Pi_{L}^{0}$ is the subspace of $\Pi_{L} \subset L^{2}(\mathcal{M}, d \mu)$ orthogonal to the constant functions, that is $\Pi_{L}^{0}=\operatorname{span}\left\{\varphi_{k}: 0<\lambda_{k} \leq L\right\}$.

By Weyl's estimates on the spectrum of an elliptic operator [13, Theorem 17.5.3], $\operatorname{dim}\left(\Pi_{L}\right) \sim L^{d}$. For each $L \geq 0$ denote with $N(L)$ the minimal number of points in an $L$-design in $\mathcal{M}$.

Proposition 1. There exists a positive constant $c_{\mathcal{M}}$ such that $N(L) \geq c_{\mathcal{M}} L^{d}$ for every $L \geq 0$.

2010 Mathematics Subject Classification. 41A55, 58J35, 42C15.

Key words and phrases. Designs, Riemannian manifolds, Marcinkiewicz-Zygmund inequalities. 
Proof. Assume $\left\{x_{j}\right\}_{j=1}^{N(L)}$ is an $L$-design with exactly $N(L)$ nodes. By Theorem 2.12 in [3] there exists a constant $\beta>0$ such that for every $f$ in the Sobolev space $W^{\alpha, 1}(\mathcal{M})$ with $\alpha>d$ one has

$$
\left|\int_{\mathcal{M}} f(x) d \mu(x)-\sum_{j=1}^{N(L)} \frac{1}{N(L)} f\left(x_{j}\right)\right| \leq \beta L^{-\alpha}\|f\|_{\alpha, 1} .
$$

On the other hand, by Theorem 2.16 in [3], there exists a constant $\gamma>0$ such that for every $L$ there exists a function $f_{L} \in W^{\alpha, 1}(\mathcal{M})$ with

$$
\left|\int_{\mathcal{M}} f_{L}(x) d \mu(x)-\sum_{j=1}^{N(L)} \frac{1}{N(L)} f_{L}\left(x_{j}\right)\right| \geq \gamma N(L)^{-\alpha / d}\left\|f_{L}\right\|_{\alpha, 1} .
$$

This gives

$$
N(L) \geq \gamma^{d / \alpha} \beta^{-d / \alpha} L^{d}
$$

Korevaar and Meyers [15] conjectured that when $\mathcal{M}$ is the $d$-dimensional sphere, there is a constant $C_{d}$ such that $N(L) \leq C_{d} L^{d}$ for any positive $L$. Bondarenko, Radchenko and Viazovska [2] show an even stronger version of Korevaar and Meyer's conjecture, namely they show that there is a constant $C_{d}$ such that for every $N \geq$ $C_{d} L^{d}$ there exists an $L$-design in the $d$-dimensional sphere with exactly $N$ nodes. Later, Etayo, Marzo and Ortega-Cerdà [8] by means of the same techniques as in 2], generalize the result of Bondarenko, Radchenko and Viazovska to the case of an affine algebraic manifold. In particular the main ingredients in these proofs are a result from the Brouwer degree theory, a partition of the ambient space $\mathcal{M}$ into equal area regions with small diameter, and a Marcinkiewicz-Zygmund inequality for the gradient of diffusion polynomials. The result from the Brouwer degree theory is completely abstract and can be directly applied independently of the manifold.

Theorem 2. 18, Theorem 1.2.9.] Let $H$ be a finite dimensional Hilbert space with inner product $\langle\cdot, \cdot\rangle$. Let $f: H \rightarrow H$ be a continuous mapping and $\Omega$ an open bounded subset with boundary $\partial \Omega$ such that $0 \in \Omega \subset H$. If $\langle x, f(x)\rangle>0$ for all $x \in \partial \Omega$, then there exists $x \in \Omega$ satisfying $f(x)=0$.

The second result has been proved recently in 11 for Ahlfors regular metric measure spaces, and therefore holds for compact Riemannian manifolds as well.

Theorem 3. There exist two positive constants $c_{1}$ and $c_{2}$ such that for every $N \geq$ 1 there is a collection of sets $\mathcal{R}=\left\{R_{j}\right\}_{j=1}^{N}$ that partition $\mathcal{M}$ in the sense that $\cup_{j=1}^{N} R_{j}=\mathcal{M}$, and $\mu\left(R_{i} \cap R_{j}\right)=0$ for all $1 \leq i<j \leq N$, such that each region has measure $1 / N$, is contained in a geodesic (closed) ball $X_{j}$ of radius $c_{2} N^{-1 / d}$ and contains a geodesic ball $Y_{j}$ of radius $c_{1} N^{-1 / d}$.

The Marcinkiewicz-Zygmund inequality for diffusion polynomials on manifolds (and much more general spaces) has been proved in a series of papers by Maggioni, Mhaskar and Filbir [9, 10, 17].

Theorem 4. [10, Theorem 5.1] Assume that $c_{1}$ and $c_{2}$ are constants for which Theorem 3 holds. Then there exists a constant $C>0$ such that for all integers $N \geq 1$, for all partitions $\mathcal{R}=\left\{R_{j}\right\}_{j=1}^{N}$ with constants $c_{1}$ and $c_{2}$ as in Theorem 3 , for all $x_{j} \in R_{j}$, for all $L \leq N^{1 / d}$ and for all $P \in \Pi_{L}$

$$
\left|\int_{\mathcal{M}}\right| P(x)\left|d \mu(x)-\sum_{j=1}^{N} \frac{1}{N}\right| P\left(x_{j}\right)|| \leq C L N^{-1 / d} \int_{\mathcal{M}}|P(x)| d \mu(x) .
$$


When $\mathcal{M}$ is the sphere and the diffusion polynomials are restrictions to $\mathcal{M}$ of polynomials in $d+1$ real variables of degree at most $L$, then the gradient of a polynomial is again a polynomial, and therefore the Marcinkiewicz-Zygmund inequality for gradients follows easily from the above Theorem 4 . In the case of algebraic manifolds, ad hoc arguments that use the complexification of the variety $\mathcal{M}$ can be applied (see [8]). In the general case of Riemannian manifolds, the above types of arguments fail. Here we show a Marcinkiewicz-Zygmund inequality for gradients of diffusion polynomials and consequently prove the Korevaar and Meyer's conjecture in the case of Riemannian manifolds.

Theorem 5. Assume that $c_{1}$ and $c_{2}$ are constants for which Theorem 3 holds. Then there exists a constant $C_{3}=C_{3}\left(c_{1}, c_{2}\right)>0$ such that for all integers $N \geq 1$, for all partitions $\mathcal{R}=\left\{R_{j}\right\}_{j=1}^{N}$ with constants $c_{1}$ and $c_{2}$ as in Theorem 3 , for all $x_{j} \in R_{j}$, for all $L \leq N^{1 / d}$ and for all $P \in \Pi_{L}^{0}$,

$$
\left|\int_{\mathcal{M}}\|\nabla P(x)\| d \mu(x)-\sum_{j=1}^{N} \frac{1}{N}\left\|\nabla P\left(x_{j}\right)\right\|\right| \leq C_{3} L N^{-1 / d} \int_{\mathcal{M}}\|\nabla P(x)\| d \mu(x) .
$$

Theorem 6. There exists a constant $C_{\mathcal{M}}$ such that for each $N \geq C_{\mathcal{M}} L^{d}$ there exists an L-design in $\mathcal{M}$ with $N$ nodes.

In the proof of Proposition 1 we mentioned Theorem 2.12 in [3. This is a result on numerical integration for functions in Sobolev spaces and it says that if $\left\{x_{j}\right\}_{j=1}^{N}$ is an $L$-design on a compact Riemannian manifold $\mathcal{M}$, then for every $1 \leq p \leq+\infty$ and for every $\alpha>d / p$ there exists a constant $\beta>0$ such that for every $f$ in the Sobolev space $W^{\alpha, p}(\mathcal{M})$

$$
\left|\int_{\mathcal{M}} f(x) d \mu(x)-\frac{1}{N} \sum_{j=1}^{N} f\left(x_{j}\right)\right| \leq \beta L^{-\alpha}\|f\|_{\alpha, p}
$$

By the above Theorem 6, if $\mathcal{M}$ is a connected compact oriented $d$-dimensional Riemannian manifold, for every positive integer $N$, setting $L=\left(N / C_{\mathcal{M}}\right)^{1 / d}$ there indeed exists an $L$-design on $\mathcal{M}$ consisting of $N$ nodes, and this immediately gives the following result on the worst case error in numerical integration

Corollary 7. For every $1 \leq p \leq+\infty$ and for every $\alpha>d / p$ there exists a constant $\beta>0$ such that for every $N \geq 1$ there exists a collection of points $\left\{x_{j}\right\}_{j=1}^{N}$ such that for every $f \in W^{\alpha, p}(\mathcal{M})$

$$
\left|\int_{\mathcal{M}} f(x) d \mu(x)-\frac{1}{N} \sum_{j=1}^{N} f\left(x_{j}\right)\right| \leq \beta C_{\mathcal{M}}^{\alpha / d} N^{-\alpha / d}\|f\|_{\alpha, p} .
$$

By Theorem 2.16 in 3 , the exponent $-\alpha / d$ is best possible. This result should be compared with Corollary 6.3, Corollary 6.4 and Example 6.5 in [4, where the authors prove that if $1<p \leq+\infty$ and $d / p<\alpha<d$, then a random choice of nodes $x_{j} \in R_{j}$ gives the desired decay rate $N^{-\alpha / d}$ for the worst case error in numerical integration if and only if $\alpha<d / 2+1$. See also [5, 6] for previous results in the case of the sphere.

We wish to thank Luca Brandolini, Leonardo Colzani and Giancarlo Travaglini for several discussions on this subject.

\section{IntRoduction to RIEMANNIAN MANIFOLDS}

The following are well known facts about manifolds. The interested reader can find all details in [1, 7]. 
A differentiable manifold of dimension $d$ is a set $\mathcal{M}$ and a family of injective maps $x_{\alpha}: U_{\alpha} \subset \mathbb{R}^{d} \rightarrow \mathcal{M}$ such that

(1) The $U_{\alpha}$ 's are open and $\cup_{\alpha} x_{\alpha}\left(U_{\alpha}\right)=\mathcal{M}$.

(2) For any pair $\alpha, \beta$ with $x_{\alpha}\left(U_{\alpha}\right) \cap x_{\beta}\left(U_{\beta}\right)=W \neq \emptyset$, the sets $x_{\alpha}^{-1}(W)$ and $x_{\beta}^{-1}(W)$ are open and the maps $x_{\beta}^{-1} \circ x_{\alpha}$ are $\mathcal{C}^{\infty}$.

(3) The family $\left\{\left(U_{\alpha}, x_{\alpha}\right)\right\}$ is maximal relative to the above conditions.

Each $\left(U_{\alpha}, x_{\alpha}\right)$ is called local chart, and a family $\left\{\left(U_{\alpha}, x_{\alpha}\right)\right\}$ satisfying (1) and (2) is called differentiable structure.

This induces a natural topology on $\mathcal{M}$. A set $A$ is open in $\mathcal{M}$ if and only if $x_{\alpha}^{-1}\left(A \cap x_{\alpha}\left(U_{\alpha}\right)\right)$ is open in $\mathbb{R}^{d}$ for all $\alpha$. We will assume that with this topology $\mathcal{M}$ is a Hausdorff space with a countable basis.

We also say that a differentiable manifold $\mathcal{M}$ is orientable if

(4) For every pair $\alpha, \beta$ with $x_{\alpha}\left(U_{\alpha}\right) \cap x_{\beta}\left(U_{\beta}\right)=W \neq \emptyset$ the differential of the change of coordinates $x_{\beta}^{-1} \circ x_{\alpha}$ has positive determinant.

A map $f: \mathcal{N} \rightarrow \mathcal{M}$ between two differentiable manifolds is called differentiable in $p \in \mathcal{N}$ if for every local chart $(V, y)$ at $f(p)$ there exists a local chart $(U, x)$ at $p$ such that $f(x(U)) \subset y(V)$ and the map $y^{-1} \circ f \circ x$ is $\mathcal{C}^{\infty}$. We say that $f$ is differentiable in an open set of $\mathcal{N}$ if it is differentiable at all points of this open set.

A differentiable map $\alpha$ from the interval $(-\varepsilon, \varepsilon) \subset \mathbb{R}$ to $\mathcal{M}$ will be called a (differentiable) curve in $\mathcal{M}$. Suppose that $\alpha(0)=p$. Call $\mathcal{D}(\mathcal{M})$ the set of functions on $\mathcal{M}$ differentiable in $p$. The tangent vector to $\alpha$ at $t=0$ is the function $\alpha^{\prime}(0)$ : $\mathcal{D}(\mathcal{M}) \rightarrow \mathbb{R}$ given by

$$
\alpha^{\prime}(0) f=\left.\frac{d(f \circ \alpha)}{d t}\right|_{t=0}, \quad f \in \mathcal{D}(\mathcal{M}) .
$$

A tangent vector at $p$ is the tangent vector at $t=0$ of a1 curve like the one above. The set of all tangent vectors to $\mathcal{M}$ at $p$ will be indicated with $T_{p} \mathcal{M}$. This is a vector space of dimension $d$ called tangent space of $\mathcal{M}$ at $p$, and the choice of a local chart $(U, x)$ around $p$ determines a natural basis of $T_{p} \mathcal{M}$ given by

$$
\left\{\left(\frac{\partial}{\partial x_{i}}\right)_{p}\right\}_{i=1}^{d}
$$

where setting $x^{0}=x^{-1}(p),\left(\partial / \partial x_{i}\right)_{p}$ is the tangent vector to the curve $x\left(x_{1}^{0}, \ldots, x_{i}^{0}+\right.$ $\left.t, \ldots, x_{d}^{0}\right)$ at $t=0$. The set $T \mathcal{M}=\left\{(p, v): p \in \mathcal{M}, v \in T_{p} \mathcal{M}\right\}$ can be given a differentiable structure that makes it a differentiable manifold of dimension $2 d$ called tangent bundle. The local charts are $\left(U_{\alpha} \times \mathbb{R}^{d}, y_{\alpha}\right)$, where

$$
y_{\alpha}\left(x_{1}^{\alpha}, \ldots, x_{d}^{\alpha}, u_{1}, \ldots, u_{d}\right)=\left(x_{\alpha}\left(x_{1}^{\alpha}, \ldots, x_{d}^{\alpha}\right), \sum_{i=1}^{d} u_{i} \frac{\partial}{\partial x_{i}^{\alpha}}\right) .
$$

A vector field $X$ on a differentiable manifold $\mathcal{M}$ is a map from $\mathcal{M}$ to the tangent bundle $T \mathcal{M}$ such that $X(p)=(p, v)$ for some $v \in T_{p} \mathcal{M}$. We call $\mathcal{X}(\mathcal{M})$ the space of differentiable vector fields on $\mathcal{M}$.

A Riemannian metric on a differentiable manifold $\mathcal{M}$ is a correspondence which associates to each point $p \in \mathcal{M}$ an inner product $\langle\cdot, \cdot\rangle_{p}$ (that is, a symmetric, bilinear, positive definite form) on the tangent space $T_{p} \mathcal{M}$ which varies differentiably in the sense that if $(U, x)$ is a local chart around $p$ and if $q=x\left(x_{1}, \ldots, x_{d}\right)$ then

$$
g_{i j}\left(x_{1}, \ldots, x_{d}\right)=\left\langle\left(\frac{\partial}{\partial x_{i}}\right)_{q},\left(\frac{\partial}{\partial x_{j}}\right)_{q}\right\rangle_{q}
$$

is a differentiable function on $U$. A differentiable manifold with a given Riemannian metric will be called a Riemannian manifold. If $v$ is a tangent vector to $\mathcal{M}$ at $p$, 
we set $\|v\|_{p}^{2}=\langle v, v\rangle_{p}$. The length of a differentiable curve $\alpha$ from the interval $[a, b]$ to $\mathcal{M}$ is defined as

$$
\int_{a}^{b}\left\|\alpha^{\prime}(t)\right\|_{\alpha(t)} d t
$$

We define the distance $|p-q|$ between two points in a Riemannian manifold, $p, q \in \mathcal{M}$, as the infimum of the lengths of all the differentiable curves joining $p$ and $q$. This is indeed a distance, and it turns $\mathcal{M}$ into a metric space that has the same topology as the manifold's natural topology.

If $\mathcal{M}$ is a compact Riemannian manifold, then for any two points $p$ and $q$ in $\mathcal{M}$ there exists at least one differentiable curve $\alpha$ joining $p$ and $q$ that realizes the infimum of the lengths of all the differentiable curves joining $p$ and $q$. Furthermore, the covariant derivative of $\alpha^{\prime}$ along $\alpha$ equals zero (curves that satisfy this property are called geodesics). We refer the reader to [7] for the precise definition of covariant derivative, here it suffices to recall that if $\alpha$ is a geodesic then $\left\|\alpha^{\prime}(t)\right\|_{\alpha(t)}$ is constant, and one can normalize $\alpha$ in such a way that $\left\|\alpha^{\prime}(t)\right\|_{\alpha(t)}=1$.

Let $(U, x)$ be a local chart where the metric has local representation given by $g_{i j}\left(x_{1}, \ldots, x_{d}\right)$. The positive measure $d \nu_{(U, x)}=\sqrt{\operatorname{det}\left(g_{i j}\right)} d x_{1} \ldots d x_{d}$ on $U$ induces a positive measure $d \mu_{(U, x)}$ on $x(U)$ given by

$$
\int_{x(U)} f d \mu_{(U, x)}=\int_{U} f \circ x d \nu_{(U, x)} .
$$

If $(U, x)$ and $(V, y)$ are two local charts with $U \cap V=W \neq \emptyset$, then one can show that $d \mu_{(U, x)}$ coincides with $d \mu_{(V, y)}$ on $W$. By a standard partition of unit argument, there exists a unique measure $d \mu$ on $\mathcal{M}$ that coincides with $d \mu_{(U, x)}$ on $U$ for all local charts $(U, x)$. This measure is called canonical measure on $\mathcal{M}$.

It can be shown that there exist two positive constants $c_{4}$ and $c_{5}$ such that for any point $x \in \mathcal{M}$ and for any radius $r \leq \operatorname{diam}(\mathcal{M})$, the measure of the ball $B(x, r)=\{y \in \mathcal{M}:|x-y|<r\}$ satisfies the inequalities

$$
c_{4} r^{d} \leq \mu(B(x, r)) \leq c_{5} r^{d}
$$

It follows easily that there exists a positive constant $c_{6}$ such that if $f:[0,+\infty) \rightarrow$ $[0,+\infty)$ is a decreasing function and if $x \in \mathcal{M}$ then

$$
\int_{\mathcal{M}} f(|x-y|) d \mu(y) \leq c_{6} \int_{0}^{+\infty} f(t) t^{d-1} d t .
$$

For any $f \in \mathcal{D}(\mathcal{M})$ we define the gradient of $f$ as a vector field $\nabla f$ on the Riemannian manifold $\mathcal{M}$ given by

$$
\langle\nabla f(p), v\rangle_{p}=v f, \quad p \in \mathcal{M}, v \in T_{p} \mathcal{M} .
$$

In local coordinates, the gradient is given by the formula

$$
\nabla f(p)=\sum_{j=1}^{d} \sum_{i=1}^{d} g^{i j}\left(\frac{\partial}{\partial x_{i}} f\right) \frac{\partial}{\partial x_{j}}
$$

where $g^{i j}$ are the entries of the inverse matrix of $g_{i j}$. It follows from the definition that

$$
\|\nabla f\|_{p}=\sup X f
$$

where the supremum is taken over all the differentiable vector fields $X$ with norm $\|X\|_{p} \leq 1$. then

If $f \in \mathcal{D}(\mathcal{M})$ and $\alpha:[0,|p-q|] \rightarrow \mathcal{M}$ is a normalized geodesic joining $p$ and $q$,

$$
|f(p)-f(q)|=\left|\int_{0}^{|p-q|} \frac{d}{d t}(f(\alpha(t))) d t\right|=\left|\int_{0}^{|p-q|}\left\langle\nabla f(\alpha(t)), \alpha^{\prime}(t)\right\rangle d t\right|
$$




$$
\leq|p-q| \sup _{t}\|\nabla f(\alpha(t))\| .
$$

One can also define the divergence of a differentiable vector field $X$. To keep this presentation simple, we only give the definition in local coordinates, that is

$$
\operatorname{div}(X)=\frac{1}{\sqrt{\operatorname{det}\left(g_{i j}\right)}} \sum_{k=1}^{d} \frac{\partial}{\partial x_{k}}\left(\sqrt{\operatorname{det}\left(g_{i j}\right)} X_{k}\right), \quad X=\sum_{k=1}^{d} X_{k} \frac{\partial}{\partial x_{k}} .
$$

Finally, the Laplace-Beltrami operator $\triangle: \mathcal{D}(\mathcal{M}) \rightarrow \mathcal{D}(\mathcal{M})$ is defined as

$$
\triangle f=-\operatorname{div}(\nabla f) \text {. }
$$

Let $\mathcal{M}$ be a compact oriented Riemannian manifold and let $f \in \mathcal{D}(\mathcal{M})$ and $X$ a differentiable vector field. The following Green identity (see [16, page 267]) holds

$$
\int_{\mathcal{M}}\langle\nabla f, X\rangle d \mu=-\int_{\mathcal{M}} f \operatorname{div}(X) d \mu .
$$

Let now $\mathcal{M}$ be a connected compact Riemannian manifold. The Laplace-Beltrami operator $\triangle$ is self adjoint and positive definite. The eigenvalues of the LaplaceBeltrami operator are the values $\lambda^{2}$ such that the equation

$$
\triangle f=\lambda^{2} f
$$

has a solution $f \in \mathcal{D}(\mathcal{M}), f \neq 0$, called eigenfunction. These eigenvalues form a discrete sequence of non-negative numbers diverging to $+\infty$. The eigenfunctions associated with a given eigenvalue $\lambda^{2}$ form a finite dimensional subspace of $\mathcal{D}(\mathcal{M})$ called eigenspace associated with $\lambda^{2}$. The dimension of the eigenspace will be called multiplicity of the eigenvalue. Different eigenspaces are orthogonal to each other in the Hilbert space $L^{2}(\mathcal{M}, d \mu)$, and the direct sum of all the eigenspaces is dense in $\mathcal{D}(\mathcal{M})$ in the topology of the uniform convergence (and, a fortiori, in $L^{2}(\mathcal{M}, d \mu)$ ). The value $\lambda^{2}=0$ is an eigenvalue and the associated eigenspace is the 1-dimensional space consisting of the constant functions on $\mathcal{M}$. It is therefore convenient to list the eigenvalues as a non decreasing sequence

$$
\lambda_{0}^{2}=0<\lambda_{1}^{2} \leq \lambda_{2}^{2} \leq \ldots
$$

where the repetitions correspond to eigenvalues with multiplicity greater than 1 . We can therefore associate each eigenvalue $\lambda_{k}^{2}$ with an eigenfunction $\varphi_{k}$ in such a way that $\left\{\varphi_{k}\right\}_{k=0}^{+\infty}$ forms an orthonormal basis of $L^{2}(\mathcal{M}, d \mu)$.

We conclude this section with a result concerning certain estimates on the derivatives of the heat kernel. See [12, Theorem 1.4.3 and the following remarks], and [14, Theorem 5.5] for an extension to general elliptic operators on manifolds of bounded geometry. See also [1, Chapter 3.E] for details on the Minakshisundaram-Pleijel asymptotic development of the heat kernel.

Theorem 8. Let $\mathcal{M}$ be a compact Riemannian manifold of dimension $d$. Let $X_{1}, \ldots, X_{\ell}$ and $Y_{1}, \ldots, Y_{m}$ be differentiable vector fields on $\mathcal{M}$ such that $\left\|X_{j}\right\|_{x} \leq 1$ and $\left\|Y_{i}\right\|_{x} \leq 1$ for all $x \in \mathcal{M}$, for all $j=1, \ldots, \ell$ and for all $i=1, \ldots, m$. Then there exist two positive constants $c_{7}=c_{7}(\ell, m)$ and $c_{8}=c_{8}(\ell, m)$ depending only on $\ell, m$ (and on $\mathcal{M}$ ) such that for all $t \in[0,1]$ and for all $x, y \in \mathcal{M}$,

$$
\left|\sum_{k=0}^{+\infty} \exp \left(-\lambda_{k}^{2} t\right) X_{1} \ldots X_{\ell} \varphi_{k}(x) Y_{1} \ldots Y_{m} \varphi_{k}(y)\right| \leq c_{7} t^{-\frac{d+\ell+m}{2}} \exp \left(-c_{8} \frac{|x-y|^{2}}{t}\right) .
$$




\section{Estimates ON SUMMABILITY KERNELS}

Here we recall certain definitions and results concerning general summability kernels for Bessel systems, following [9].

Definition 9. A system $\left\{\phi_{k}\right\}_{k=0}^{+\infty} \subset L^{2}(\mathcal{M})$ will be called a generalized Bessel system if for any $g \in \mathcal{D}(\mathcal{M})$,

$$
\mathcal{N}(g):=\sum_{k=0}^{+\infty}\left|\int_{\mathcal{M}} g \phi_{k} d \mu\right|^{2}<+\infty .
$$

Any Bessel system, that is a system $\left\{\phi_{k}\right\}_{k=0}^{+\infty}$ such that for all $f \in L^{2}(\mathcal{M})$ one has $\sum_{k=0}^{+\infty}\left|\int_{\mathcal{M}} f \phi_{k} d \mu\right|^{2} \leq c\|f\|_{2}^{2}$, is clearly a generalized Bessel system according to the above definition. In particular, any orthonormal system is a generalized Bessel system. We will also use the following type of generalized Bessel systems. Let $X$ be a differentiable vector field on $\mathcal{M}$ and set $\phi_{k}=X \varphi_{k}$, where $\left\{\varphi_{k}\right\}_{k=0}^{+\infty}$ are the eigenfunctions of the Laplace-Beltrami operator described before. By Green's formula (4), for any $g \in \mathcal{D}(\mathcal{M})$

$$
\sum_{k=0}^{+\infty}\left|\int_{\mathcal{M}} g\left(X \varphi_{k}\right) d \mu\right|^{2}=\sum_{k=0}^{+\infty}\left|-\int_{\mathcal{M}} \operatorname{div}(g X) \varphi_{k} d \mu\right|^{2}=\|\operatorname{div}(g X)\|_{2}^{2}<+\infty .
$$

Similarly, if $X_{1}, X_{2}$ are differentiable vector fields on $\mathcal{M}$ and we set $\phi_{k}=X_{1} X_{2} \varphi_{k}$, then

$$
\sum_{k=0}^{+\infty}\left|\int_{\mathcal{M}} g\left(X_{1} X_{2} \varphi_{k}\right) d \mu\right|^{2}=\left\|\operatorname{div}\left(\operatorname{div}\left(g X_{1}\right) X_{2}\right)\right\|_{2}^{2}<+\infty
$$

and so on for any number of vector fields.

Here is the main result of this section.

Theorem 10 (Theorem 2.1 in 9]). Let $\left\{\phi_{k}\right\},\left\{\psi_{k}\right\}$ be generalized Bessel systems composed by continuous functions, and assume that there exist positive constants $\kappa_{1}, \kappa_{2}, \kappa_{3}, \kappa_{4}, A_{1}, A_{2}, A_{3}$ such that for all $t \in(0,1]$ and $x, y \in \mathcal{M}$

$$
\begin{aligned}
\sum_{k=0}^{+\infty} \exp \left(-\lambda_{k}^{2} t\right)\left|\phi_{k}(x)\right|^{2} & \leq \kappa_{1} t^{-A_{1} / 2}, \\
\sum_{k=0}^{+\infty} \exp \left(-\lambda_{k}^{2} t\right)\left|\psi_{k}(x)\right|^{2} & \leq \kappa_{2} t^{-A_{2} / 2}, \\
\left|\sum_{k=0}^{+\infty} \exp \left(-\lambda_{k}^{2} t\right) \phi_{k}(x) \psi_{k}(y)\right| & \leq \kappa_{3} t^{-A_{3}} \exp \left(-\kappa_{4}|x-y|^{2} / t\right) .
\end{aligned}
$$

Let $K=\left(A_{1}+A_{2}\right) / 2$ and $S>\max \{K, d\}$ integer. Let $H: \mathbb{R} \rightarrow \mathbb{R}$ be an even function supported on $[-1,1]$ with continuous derivatives up to order $S$. Then there exists a positive constant $C_{9}$ such that for all $x, y \in \mathcal{M}$ and for all $L>0$,

$$
\left|\sum_{k=0}^{+\infty} H\left(\lambda_{k} / L\right) \phi_{k}(x) \psi_{k}(y)\right| \leq C_{9} \frac{L^{K}}{(1+L|x-y|)^{S}} .
$$

The constant $C_{9}$ does not depend on the specific Bessel systems $\left\{\phi_{k}\right\}$ and $\left\{\psi_{k}\right\}$ nor on the manifold $\mathcal{M}$, but only on the constants $\kappa_{1}, \kappa_{2}, \kappa_{3}, \kappa_{4}, A_{1}, A_{2}, A_{3}$, and on the function $H$.

In particular, assume that the generalized Bessel systems are given by

$$
\phi_{k}=X_{1} \ldots X_{\ell} \varphi_{k}, \quad \psi_{k}=Y_{1} \ldots Y_{m} \varphi_{k}
$$

where $X_{1}, \ldots, X_{\ell}$ and $Y_{1}, \ldots, Y_{m}$ are differentiable vector fields on $\mathcal{M}$ such that $\left\|X_{j}\right\|_{x} \leq 1$ and $\left\|Y_{i}\right\|_{x} \leq 1$ for all $x \in \mathcal{M}$, for all $j=1, \ldots, \ell$ and for all $i=$ 
$1, \ldots, m$. Then, by Theorem 8 , the above Theorem 10 applies with $\kappa_{1}=c_{7}(\ell, \ell)$, $\kappa_{2}=c_{7}(m, m), \kappa_{3}=c_{7}(\ell, m), \kappa_{4}=c_{8}(\ell, m), A_{1}=d+2 \ell, A_{2}=d+2 m$, and $A_{3}=(d+\ell+m) / 2$.

Theorem 10 follows from a series of results, the first being Theorem 4.1 again in 9 . The latter is a nice elementary result on holomorphic functions, which is a simplified version of a result of A. Sikora [19, Theorem 2]. Unfortunately, the proof of Theorem 4.1 presented in [9] is incomplete, as inequality (4.12) is not properly justified. Actually one needs to use, as A. Sikora does in his original proof, some version of the Phragmén-Lindelöf theorem. We give here a complete proof of this result.

Theorem 11 (Theorem 4.1 in [9]). Let $r>0,\left\{a_{k}\right\}$ be an absolutely summable sequence of complex numbers, $\left\{\ell_{k}\right\}$ be a sequence of nonnegative, nondecreasing numbers with $\ell_{k} \rightarrow \infty$, and

$$
K(t)=\sum_{k=0}^{\infty} \exp \left(-\ell_{k}^{2} t\right) a_{k}, \quad W(t)=\sum_{k=0}^{\infty} \cos \left(\ell_{k} t\right) a_{k} .
$$

Then

$$
|K(t)| \leq \alpha t^{-\beta} \exp \left(-r^{2} / t\right) \sum_{k=0}^{\infty}\left|a_{k}\right|, \quad t \in(0,1]
$$

if and only if $W(t)=0$ for $0 \leq t \leq 2 r$.

Proof. Assume without loss of generality that $\sum_{k=0}^{\infty}\left|a_{k}\right|=1$. By the well known formula on the Fourier transform of the Gaussian function, for all $t>0$ and for all $k=0,1,2, \ldots$

$$
\exp \left(-\ell_{k}^{2} t\right)=\frac{1}{\sqrt{\pi t}} \int_{0}^{+\infty} \exp \left(-u^{2} /(4 t)\right) \cos \left(\ell_{k} u\right) d u
$$

and, by the absolute and uniform convergence of the series defining $W(t)$,

$$
K(t)=\frac{1}{\sqrt{\pi t}} \int_{0}^{+\infty} \exp \left(-u^{2} /(4 t)\right) W(u) d u
$$

Assume now $W(u)=0$ for all $0 \leq u \leq 2 r$. If $t \geq r^{2}$ then

$$
|K(t)| \leq 1 \leq \exp \left(1-r^{2} / t\right)=e \exp \left(-r^{2} / t\right) .
$$

If $t<r^{2}$ then, by (5),

$$
\begin{aligned}
|K(t)| & \leq \frac{1}{\sqrt{\pi t}} \int_{2 r}^{+\infty} \exp \left(-u^{2} /(4 t)\right)|W(u)| d u \\
& \leq \frac{1}{\sqrt{\pi t}} \int_{2 r}^{+\infty} \exp \left(-u^{2} /(4 t)\right) d u \\
& =\frac{1}{\sqrt{\pi}} \int_{r^{2} / t}^{+\infty} u^{-1 / 2} \exp (-u) d u \\
& \leq \frac{1}{\sqrt{\pi}}\left(\frac{t}{r^{2}}\right)^{1 / 2} \exp \left(-\frac{r^{2}}{t}\right) \leq \frac{1}{\sqrt{\pi}} \exp \left(-\frac{r^{2}}{t}\right)
\end{aligned}
$$

and the thesis follows with $\alpha=e$ and $\beta=0$.

Assume now that

$$
|K(t)| \leq \alpha t^{-\beta} \exp \left(-r^{2} / t\right), \quad t \in(0,1] .
$$

Then for any $\varepsilon>0$ there exists a positive constant $\gamma$ such that

$$
|K(t)| \leq \gamma \exp \left(-(r-\varepsilon)^{2} / t\right), \quad t \in(0,1] .
$$


For any complex $t=\tau+i \xi$ with $\tau \geq 0$, define the function

$$
F(t)= \begin{cases}\frac{t}{1+t} \exp \left(4(r-\varepsilon)^{2} t\right) \sum_{k=0}^{+\infty} \exp \left(-\ell_{k}^{2} /(4 t)\right) a_{k} & t \neq 0, \tau \geq 0 \\ 0 & t=0 .\end{cases}
$$

It is easy to show that $F$ satisfies the hypotheses of the Phragmén-Lindelöf theorem (see [20, Theorem 3.4 page 124]) in $\left\{-\frac{\pi}{2}<\arg (t)<0\right\}$ and in $\left\{0<\arg (t)<\frac{\pi}{2}\right\}$. In particular, by (6), for all $\tau>0$

$$
\begin{aligned}
|F(\tau)| & \leq \begin{cases}\frac{\tau}{1+\tau} \exp \left(4(r-\varepsilon)^{2} \tau\right) \gamma \exp \left(-(r-\varepsilon)^{2} 4 \tau\right) & \text { if } 1 /(4 \tau) \leq 1 \\
\frac{\tau}{1+\tau} \exp \left(4(r-\varepsilon)^{2} \tau\right) & \text { if } 1 /(4 \tau) \geq 1\end{cases} \\
& \leq \begin{cases}\gamma & \text { if } \tau \geq 1 / 4 \\
\frac{1}{5} \exp \left((r-\varepsilon)^{2}\right) & \text { if } \tau \leq 1 / 4 \\
& \leq \max \left\{\gamma, \frac{1}{5} \exp \left(r^{2}\right), 1\right\}=: \eta .\end{cases}
\end{aligned}
$$

Also, for all $\xi \in \mathbb{R} \backslash\{0\}$,

$$
|F(i \xi)| \leq \frac{|\xi|}{|1+i \xi|} \sum_{k=0}^{+\infty}\left|a_{k}\right| \leq 1 \leq \eta .
$$

The function $F$ is continuous on $\{\operatorname{Re}(t) \geq 0\}$. The continuity outside 0 follows by the absolute and uniform convergence of the series defining $F$ in any compact subset of $\{\operatorname{Re}(t) \geq 0\} \backslash\{0\}$. The continuity in 0 follows by the estimate, for $\operatorname{Re}(t) \geq 0$ and $0<|t|<\delta$,

$$
\begin{aligned}
|F(t)| & \leq|t| \exp \left(4(r-\varepsilon)^{2} \delta\right) \sum_{k=0}^{+\infty} \exp \left(-\ell_{k}^{2} \tau /\left(4|t|^{2}\right)\right)\left|a_{k}\right| \\
& \leq|t| \exp \left(4(r-\varepsilon)^{2} \delta\right)
\end{aligned}
$$

Again by the absolute and uniform convergence of the series defining $F$, it follows that $F$ is holomorphic in $\{\operatorname{Re}(t)>0\}$. Finally, for all $\operatorname{Re}(t)>0$,

$$
\begin{aligned}
|F(t)| & \leq \exp \left(4(r-\varepsilon)^{2} \tau\right) \sum_{k=0}^{+\infty} \exp \left(-\ell_{k}^{2} \tau /\left(4\left(\tau^{2}+\xi^{2}\right)\right)\right)\left|a_{k}\right| \\
& \leq \exp \left(4(r-\varepsilon)^{2} \tau\right) \leq \exp \left(4(r-\varepsilon)^{2}|t|\right) .
\end{aligned}
$$

It therefore follows by the Phragmén-Lindelöf theorem that

$$
|F(t)| \leq \eta, \quad \forall t: \operatorname{Re}(t) \geq 0
$$

The proof now follows as in 9]. Changing variables in (5) we obtain

$$
\frac{1}{\sqrt{4 \tau}} K\left(\frac{1}{4 \tau}\right)=\int_{-\infty}^{+\infty} \exp (-u \tau) g(u) d u
$$

where

$$
g(u)= \begin{cases}\frac{1}{\sqrt{4 \pi u}} W(\sqrt{u}) & \text { if } u>0 \\ 0 & \text { if } u \leq 0\end{cases}
$$


By the definition of $F$ we have for $\tau>0$

$$
\frac{1}{\sqrt{4 \tau}} K\left(\frac{1}{4 \tau}\right)=\frac{1}{\sqrt{4 \tau}} \frac{1+\tau}{\tau} \exp \left(-4(r-\varepsilon)^{2} \tau\right) F(\tau),
$$

which can be extended analytically to $\{\operatorname{Re}(t)>0\}$. Also $\int_{-\infty}^{+\infty} \exp (-u \tau) g(u) d u$ can be extended analytically to $\{\operatorname{Re}(t)>0\}$, and the identity

$$
\frac{1}{(4 t)^{1 / 2}} \frac{1+t}{t} \exp \left(-4(r-\varepsilon)^{2} t\right) F(t)=\int_{-\infty}^{+\infty} \exp (-u t) g(u) d u
$$

holds in $\{\operatorname{Re}(t)>0\}$. Let now $\phi \in \mathcal{C}^{\infty}(\mathbb{R})$ with support in $[0, b]$. Then

$$
\widehat{\phi}(t)=\int_{-\infty}^{+\infty} \phi(u) e^{-i u t} d u
$$

is entire and a repeated integration by parts gives

$$
\begin{aligned}
\widehat{\phi}(-\xi+i \tau) & =\int_{-\infty}^{+\infty} \phi(u) e^{-i u(-\xi+i \tau)} d u \\
& =\frac{(-1)^{R}}{(-i(-\xi+i \tau))^{R}} \int_{-\infty}^{+\infty} \phi^{(R)}(u) e^{-i u(-\xi+i \tau)} d u \\
& =\frac{(-1)^{R}}{(\tau+i \xi)^{R}} \int_{-\infty}^{+\infty} \phi^{(R)}(u) e^{(\tau+i \xi) u} d u
\end{aligned}
$$

so that

$$
|\widehat{\phi}(-\xi+i \tau)| \leq \frac{\max \left(1, e^{\tau b}\right)}{\left(\tau^{2}+\xi^{2}\right)^{R / 2}} \int_{-\infty}^{+\infty}\left|\phi^{(R)}(u)\right| d u .
$$

Thus, for any $\tau>0$,

$$
\begin{aligned}
\int_{\mathbb{R}} g(u) \phi(u) d u \\
=\int_{\mathbb{R}} e^{-u \tau} g(u)\left\{e^{u \tau} \phi(u)\right\} d u \\
=\int_{\mathbb{R}} e^{-u \tau} g(u)\left\{\frac{1}{2 \pi} \int_{\mathbb{R}} e^{i \xi u}\left(\widehat{e^{\cdot \tau} \phi(\cdot)}\right)(\xi) d \xi\right\} d u \\
=\int_{\mathbb{R}} e^{-u \tau} g(u) \frac{1}{2 \pi} \int_{\mathbb{R}} e^{i \xi u} \int_{\mathbb{R}} e^{s \tau} \phi(s) e^{-i s \xi} d s d \xi d u \\
=\int_{\mathbb{R}} e^{-u \tau} g(u) \frac{1}{2 \pi} \int_{\mathbb{R}} e^{i \xi u} \int_{\mathbb{R}} \phi(s) e^{-i s(\xi+i \tau)} d s d \xi d u \\
=\int_{\mathbb{R}} e^{-u \tau} g(u) \frac{1}{2 \pi} \int_{\mathbb{R}} e^{-i \xi u} \int_{\mathbb{R}} \phi(s) e^{-i s(-\xi+i \tau)} d s d \xi d u \\
=\int_{\mathbb{R}} e^{-u \tau} g(u) \frac{1}{2 \pi} \int_{\mathbb{R}} e^{-i \xi u} \widehat{\phi}(-\xi+i \tau) d \xi d u .
\end{aligned}
$$

One can apply Fubini's theorem, since

$$
\left|e^{-u \tau} g(u) e^{-i \xi u} \widehat{\phi}(-\xi+i \tau)\right| \leq e^{-u \tau}|g(u)| \frac{e^{\tau b}}{\left(\tau^{2}+\xi^{2}\right)^{R / 2}} \int_{-\infty}^{+\infty}\left|\phi^{(R)}(u)\right| d u,
$$

so that by (7)

$$
\begin{aligned}
& \int_{\mathbb{R}} g(u) \phi(u) d u \\
& =\frac{1}{2 \pi} \int_{\mathbb{R}} \widehat{\phi}(-\xi+i \tau) \int_{\mathbb{R}} g(u) e^{-u(\tau+i \xi)} d u d \xi
\end{aligned}
$$




$$
=\frac{1}{2 \pi} \int_{\mathbb{R}} \widehat{\phi}(-\xi+i \tau) \frac{1}{(4(\tau+i \xi))^{1 / 2}} \frac{1+\tau+i \xi}{\tau+i \xi} e^{-4(r-\varepsilon)^{2}(\tau+i \xi)} F(\tau+i \xi) d \xi .
$$

It follows from this and (8) that

$$
\begin{aligned}
& \left|\int_{\mathbb{R}} g(u) \phi(u) d u\right| \\
& \leq \frac{\eta}{4 \pi} e^{-4(r-\varepsilon)^{2} \tau} \int_{\mathbb{R}}|\widehat{\phi}(-\xi+i \tau)| \frac{|1+\tau+i \xi|}{|\tau+i \xi|^{3 / 2}} d \xi \\
& \leq \frac{\eta}{4 \pi} e^{\left(b-4(r-\varepsilon)^{2}\right) \tau} \int_{-\infty}^{+\infty}\left|\phi^{(R)}(u)\right| d u \int_{\mathbb{R}}\left((1+\tau)^{2}+\xi^{2}\right)^{1 / 2}\left(\tau^{2}+\xi^{2}\right)^{-R / 2-3 / 4} d \xi
\end{aligned}
$$

which goes to 0 as $\tau \rightarrow+\infty$ if $b \leq 4(r-\varepsilon)^{2}$. By the arbitrarity of $\phi$ it follows that $g(u)=0$ for $0 \leq u \leq 4(r-\varepsilon)^{2}$, and by the arbitrarity of $\varepsilon>0, g(u)=0$ for $0 \leq u \leq 4 r^{2}$, so that $W(t)=0$ for $0 \leq t \leq 2 r$.

The next step in the proof of Theorem 10, again following [9], is

Corollary 12 (Corollary 4.1 in [9]). Let $G: \mathbb{R} \rightarrow \mathbb{R}$ be an even, bounded, integrable function such that the Fourier transform $\widehat{G}$ is also integrable and supported on $[-2 r, 2 r]$. In the assumptions of Theorem 11 , if

$$
|K(t)| \leq \alpha t^{-\beta} \exp \left(-r^{2} / t\right) \sum_{k=0}^{\infty}\left|a_{k}\right|, \quad t \in(0,1]
$$

then

$$
\sum_{k=0}^{+\infty} G\left(\ell_{k}\right) a_{k}=0
$$

Proof. By the Fourier inversion formula,

$$
G(u)=\frac{1}{\pi} \int_{0}^{+\infty} \widehat{G}(t) \cos (t u) d t .
$$

Thus,

by Theorem 11 ,

$$
\sum_{k=0}^{+\infty} G\left(\ell_{k}\right) a_{k}=\frac{1}{\pi} \int_{0}^{+\infty} \widehat{G}(t) W(t) d t=0
$$

Let now $V: \mathbb{R} \rightarrow \mathbb{R}$ be an even function such that $\widehat{V}$ is infinitely differentiable with $\widehat{V}(u)=1$ when $|u| \leq 1 / 2$ and $\widehat{V}(u)=0$ when $|u| \geq 1$. Then for any $Y>0$ let $H_{Y}$ be defined by

$$
\widehat{H}_{Y}(u)=\widehat{H}(u) \widehat{V}(u / Y) .
$$

Lemma 13 (Lemma 4.3 in 9, Lemma 6.1 in [17). In the assumptions of Theorem 10, there is a constant $c_{10}>0$ (depending on $\kappa_{1}, \kappa_{2}, \kappa_{3}, \kappa_{4}, A_{1}, A_{2}, A_{3}$ and on the function $H$ ) such that for all $Y \geq 1 / 2$ and for all $x, y \in \mathcal{M}$

$$
\left|\sum_{k=0}^{+\infty}\left(H\left(\lambda_{k} / L\right)-H_{Y}\left(\lambda_{k} / L\right)\right) \phi_{k}(x) \psi_{k}(y)\right| \leq c_{10} L^{K} Y^{-S} .
$$

The interested reader can find the proof in the above mentioned references.

Proof of Theorem 10. Again, we follow Filbir and Mhaskar 9, Proof of Theorem 2.1 page 646]. By the hypotheses, for all $L \geq 1$ we have

$$
\sum_{\lambda_{k} \leq L}\left|\phi_{k}(x)\right|^{2} \leq \sum_{k=0}^{+\infty} \exp \left(1-\lambda_{k}^{2} / L^{2}\right)\left|\phi_{k}(x)\right|^{2} \leq e \kappa_{1} L^{A_{1}},
$$


and similarly,

$$
\sum_{\lambda_{k} \leq L}\left|\psi_{k}(x)\right|^{2} \leq e \kappa_{2} L^{A_{2}} .
$$

Hence, by the Cauchy-Schwarz inequality

$$
\begin{aligned}
\left|\sum_{k=0}^{+\infty} H\left(\lambda_{k} / L\right) \phi_{k}(x) \psi_{k}(y)\right| & \leq \max _{t \in \mathbb{R}}|H(t)|\left(\sum_{\lambda_{k} \leq L}\left|\phi_{k}(x)\right|^{2}\right)^{1 / 2}\left(\sum_{\lambda_{k} \leq L}\left|\psi_{k}(y)\right|^{2}\right)^{1 / 2} \\
& \leq e\left(\kappa_{1} \kappa_{2}\right)^{1 / 2} \max _{t \in \mathbb{R}}|H(t)| L^{K}
\end{aligned}
$$

This proves the theorem when $L|x-y| \leq 1$. Assume now $L|x-y| \geq 1$ and let $Y:=\sqrt{\kappa_{4}} L|x-y|$. Let $f_{1}, f_{2} \in L^{1}(\mathcal{M})$ with $\left\|f_{1}\right\|_{1}=\left\|f_{2}\right\|_{1}=1$ be supported in the balls centered at $x$ and $y$ respectively, and with radii $|x-y| / 8$. For any $\varepsilon>0$ there exist two functions $g_{1}, g_{2} \in \mathcal{C}^{\infty}(\mathcal{M})$ supported in the balls centered at $x$ and $y$ respectively, and with radii $|x-y| / 4$ such that $\left\|f_{1}-g_{1}\right\|_{1}<\varepsilon$ and $\left\|f_{2}-g_{2}\right\|_{1}<\varepsilon$. Therefore, by (9) and Lemma 13 .

$$
\begin{aligned}
& \left|\sum_{k=0}^{+\infty} H\left(\lambda_{k} / L\right) \int_{\mathcal{M}} \int_{\mathcal{M}} \phi_{k}(w) \psi_{k}(z) f_{1}(w) f_{2}(z) d \mu(w) d \mu(z)\right| \\
& \leq\left|\sum_{k=0}^{+\infty} H\left(\lambda_{k} / L\right) \int_{\mathcal{M}} \int_{\mathcal{M}} \phi_{k}(w) \psi_{k}(z) f_{1}(w)\left(f_{2}(z)-g_{2}(z)\right) d \mu(w) d \mu(z)\right| \\
& +\left|\sum_{k=0}^{+\infty} H\left(\lambda_{k} / L\right) \int_{\mathcal{M}} \int_{\mathcal{M}} \phi_{k}(w) \psi_{k}(z)\left(f_{1}(w)-g_{1}(w)\right) g_{2}(z) d \mu(w) d \mu(z)\right| \\
& +\left|\sum_{k=0}^{+\infty} H\left(\lambda_{k} / L\right) \int_{\mathcal{M}} \int_{\mathcal{M}} \phi_{k}(w) \psi_{k}(z) g_{1}(w) g_{2}(z) d \mu(w) d \mu(z)\right| \\
& \leq\left|\sum_{k=0}^{+\infty} H\left(\lambda_{k} / L\right) \int_{\mathcal{M}} \int_{\mathcal{M}} \phi_{k}(w) \psi_{k}(z) g_{1}(w) g_{2}(z) d \mu(w) d \mu(z)\right| \\
& +e\left(\kappa_{1} \kappa_{2}\right)^{1 / 2} \varepsilon \max _{t \in \mathbb{R}} \mid H_{(t) \mid\left(\left\|f_{1}\right\|_{1}+\left\|g_{2}\right\|_{1}\right) L^{K}} \\
& \leq\left|\sum_{k=0}^{+\infty} H_{Y}\left(\lambda_{k} / L\right) \int_{\mathcal{M}} \int_{\mathcal{M}} \phi_{k}(w) \psi_{k}(z) g_{1}(w) g_{2}(z) d \mu(w) d \mu(z)\right|
\end{aligned}
$$

The distance between the supports of $g_{1}$ and $g_{2}$ exceeds $|x-y| / 2$ and therefore for all $t \in(0,1]$

$$
\begin{aligned}
& \left|\sum_{k=0}^{+\infty} \exp \left(-\lambda_{k}^{2} t\right) \int_{\mathcal{M}} \int_{\mathcal{M}} \phi_{k}(w) \psi_{k}(z) g_{1}(w) g_{2}(z) d \mu(w) d \mu(z)\right| \\
& \leq \kappa_{3} t^{-A_{3}} \exp \left(-\kappa_{4}|x-y|^{2} /(4 t)\right)\left\|g_{1}\right\|_{1}\left\|g_{2}\right\|_{1} .
\end{aligned}
$$

Observe that since $\left\{\phi_{k}\right\}$ and $\left\{\psi_{k}\right\}$ are generalized Bessel systems, then

$$
\sum_{k=0}^{+\infty}\left|\int_{\mathcal{M}} \int_{\mathcal{M}} \phi_{k}(w) \psi_{k}(z) g_{1}(w) g_{2}(z) d \mu(w) d \mu(z)\right| \leq\left(\mathcal{N}_{\left\{\phi_{k}\right\}}\left(g_{1}\right) \mathcal{N}_{\left\{\psi_{k}\right\}}\left(g_{2}\right)\right)^{1 / 2}
$$

We may therefore apply Corollary 12 with $r=\sqrt{\kappa_{4}}|x-y| / 2, G(u)=H_{Y}(u / L)$ and

$$
a_{k}=\int_{\mathcal{M}} \int_{\mathcal{M}} \phi_{k}(w) \psi_{k}(z) g_{1}(w) g_{2}(z) d \mu(w) d \mu(z) \text {. }
$$


By (10),

$$
\sum_{k=0}^{+\infty} H_{Y}\left(\lambda_{k} / L\right) \int_{\mathcal{M}} \int_{\mathcal{M}} \phi_{k}(w) \psi_{k}(z) g_{1}(w) g_{2}(z) d \mu(w) d \mu(z)=0 .
$$

Therefore

$$
\begin{aligned}
& \left|\sum_{k=0}^{+\infty} H\left(\lambda_{k} / L\right) \int_{\mathcal{M}} \int_{\mathcal{M}} \phi_{k}(w) \psi_{k}(z) f_{1}(w) f_{2}(z) d \mu(w) d \mu(z)\right| \\
& \leq c_{10} L^{K} Y^{-S}\left\|g_{1}\right\|_{1}\left\|g_{2}\right\|_{1}+e\left(\kappa_{1} \kappa_{2}\right)^{1 / 2} \varepsilon \max _{t \in \mathbb{R}}|H(t)|\left(\left\|f_{1}\right\|_{1}+\left\|g_{2}\right\|_{1}\right) L^{K} \\
& \leq c_{10}(1+\varepsilon)^{2} L^{K} Y^{-S}+e\left(\kappa_{1} \kappa_{2}\right)^{1 / 2} \varepsilon(2+\varepsilon) \max _{t \in \mathbb{R}}|H(t)| L^{K} .
\end{aligned}
$$

By the arbitrarity of $\varepsilon>0$ this gives

$$
\left|\sum_{k=0}^{+\infty} H\left(\lambda_{k} / L\right) \int_{\mathcal{M}} \int_{\mathcal{M}} \phi_{k}(w) \psi_{k}(z) f_{1}(w) f_{2}(z) d \mu(w) d \mu(z)\right| \leq c_{10} L^{K} Y^{-S},
$$

and by the arbitrarity of $f_{1}$ and $f_{2}$ and the continuity of $\phi_{k}$ and $\psi_{k}$,

$$
\left|\sum_{k=0}^{+\infty} H\left(\lambda_{k} / L\right) \phi_{k}(x) \psi_{k}(y)\right| \leq C_{9} L^{K} Y^{-S}
$$

\section{Proof of Theorem 5}

This proof follows the lines of the corresponding proof of Theorem 4 by Filbir and Mhaskar as found in [9, 10, properly modified to treat the case of the gradients.

Fix $\varepsilon>0$, and let $v_{\varepsilon}:[0,+\infty) \rightarrow \mathbb{R}$ be a $\mathcal{C}^{\infty}$ function such that $v_{\varepsilon}(u)=u$ for $u \geq \varepsilon, v_{\varepsilon}(u)=\varepsilon / 2$ for $u<\varepsilon / 4$ and $v_{\varepsilon}(u) \geq u$ for all $u \geq 0$. Let $P \in \Pi_{L}^{0}$ and define the differentiable vector field

$$
T(x):=\frac{\nabla P(x)}{v_{\varepsilon}(\|\nabla P(x)\|)} .
$$

Then

$$
T P(x)=\left\langle\nabla P(x), \frac{\nabla P(x)}{v_{\varepsilon}(\|\nabla P(x)\|)}\right\rangle=\frac{\|\nabla P(x)\|^{2}}{v_{\varepsilon}(\|\nabla P(x)\|)} \leq\|\nabla P(x)\|
$$

and

$$
\begin{aligned}
& \left|\int_{\mathcal{M}}\|\nabla P(x)\| d \mu(x)-\sum_{j=1}^{N} \frac{1}{N}\left\|\nabla P\left(x_{j}\right)\right\|\right| \\
& \leq\left|\int_{\mathcal{M}}(\|\nabla P(x)\|-T P(x)) d \mu(x)\right|+\left|\int_{\mathcal{M}} T P(x) d \mu(x)-\sum_{j=1}^{N} \frac{1}{N} T P\left(x_{j}\right)\right| \\
& +\left|\sum_{j=1}^{N} \frac{1}{N}\left(T P\left(x_{j}\right)-\left\|\nabla P\left(x_{j}\right)\right\|\right)\right| \\
& \leq 2 \varepsilon+\left|\int_{\mathcal{M}} T P(x) d \mu(x)-\sum_{j=1}^{N} \frac{1}{N} T P\left(x_{j}\right)\right| .
\end{aligned}
$$


Let us now call $\delta$ the maximum diameter of the balls $X_{j}$, so that $\delta \leq 2 c_{2} N^{-1 / d}$. Now

$$
\begin{aligned}
\left|\int_{\mathcal{M}} T P(x) d \mu(x)-\sum_{j=1}^{N} \frac{1}{N} T P\left(x_{j}\right)\right| & \leq \sum_{j=1}^{N} \int_{R_{j}}\left|T P(x)-T P\left(x_{j}\right)\right| d \mu(x) \\
& \leq \sum_{j=1}^{N} \frac{1}{N} \sup _{x, z \in R_{j}}|T P(x)-T P(z)|
\end{aligned}
$$

By (3), the last term can be bounded above by

$$
\sum_{j=1}^{N} \frac{1}{N} \sup _{x, z \in R_{j}} \sup _{t \in[0,|x-y|]}\|\nabla T P(\alpha(t))\||x-z| .
$$

where $\alpha$ is a normalized geodesic joining $x$ and $z$. Since $R_{j}$ is contained in the ball $X_{j}$, the geodesic $\alpha$ is contained in the ball $2 X_{j}$ with the same center as $X_{j}$ and radius twice the radius of $X_{j}$. It follows that the last term is bounded above by

$$
\delta \sum_{j=1}^{N} \frac{1}{N} \sup _{x \in 2 X_{j}}\|\nabla T P(x)\| .
$$

Defining now the vector field

$$
S(x):=\frac{\nabla T P(x)}{v_{\varepsilon}(\|\nabla T P(x)\|)}
$$

we have as before

$$
\operatorname{STP}(x)=\frac{\|\nabla T P(x)\|^{2}}{v_{\varepsilon}(\|\nabla T P(x)\|)} \leq\|\nabla T P(x)\|
$$

and therefore

$$
\begin{aligned}
& \delta \sum_{j=1}^{N} \frac{1}{N} \sup _{x \in 2 X_{j}}\|\nabla T P(x)\| \\
& \leq \delta \sum_{j=1}^{N} \frac{1}{N} \sup _{x \in 2 X_{j}}|\|\nabla T P(x)\|-S T P(x)|+\delta \sum_{j=1}^{N} \frac{1}{N} \sup _{x \in 2 X_{j}}|S T P(x)| \\
& \leq \delta \varepsilon+\delta \sum_{j=1}^{N} \frac{1}{N} \sup _{x \in 2 X_{j}}|S T P(x)| .
\end{aligned}
$$

So far we have obtained the inequality

$$
\left|\int_{\mathcal{M}}\|\nabla P(x)\| d \mu(x)-\sum_{j=1}^{N} \frac{1}{N}\left\|\nabla P\left(x_{j}\right)\right\|\right| \leq(2+\delta) \varepsilon+\delta \sum_{j=1}^{N} \frac{1}{N} \sup _{x \in 2 X_{j}}|\operatorname{STP}(x)| .
$$

Let $h$ be a $\mathcal{C}^{\infty}$ even function such that $h(u)$ equals 1 for $u \in[-1,1]$ and $h(u)$ equals 0 for $|u| \geq 2$. For any $L \geq 0$, define the kernels

$$
\begin{aligned}
& W_{L}(x, y)=\sum_{0<\lambda_{k}} \frac{1}{\lambda_{k}^{2}} h\left(\frac{\lambda_{k}}{L}\right) \varphi_{k}(x) \varphi_{k}(y) \\
& \Psi_{L}(x, y)=\Delta_{y} W_{L}(x, y)=\sum_{0<\lambda_{k}} h\left(\frac{\lambda_{k}}{L}\right) \varphi_{k}(x) \varphi_{k}(y) .
\end{aligned}
$$

Since $\Psi_{L}(x, y)$ is a reproducing kernel for $\Pi_{L}^{0}$, we have by Green's formula (4)

$$
P(x)=\int_{\mathcal{M}} P(y) \Psi_{L}(x, y) d \mu(y)=\int_{\mathcal{M}} P(y) \Delta_{y} W_{L}(x, y) d \mu(y)
$$




$$
=\int_{\mathcal{M}}\left\langle\nabla_{y} P(y), \nabla_{y} W_{L}(x, y)\right\rangle d \mu(y) .
$$

Thus

$$
\operatorname{STP}(x)=\int_{\mathcal{M}}\left\langle\nabla_{y} P(y), \nabla_{y} S_{x} T_{x} W_{L}(x, y)\right\rangle d \mu(y)
$$

and

$$
\begin{aligned}
|S T P(x)| & =\left|\int_{\mathcal{M}}\left\langle\nabla_{y} P(y), \nabla_{y} S_{x} T_{x} W_{L}(x, y)\right\rangle d \mu(y)\right| \\
& \leq \int_{\mathcal{M}}\left\|\nabla_{y} P(y)\right\|\left\|\nabla_{y} S_{x} T_{x} W_{L}(x, y)\right\| d \mu(y) .
\end{aligned}
$$

We will show in a moment that

$$
\left\|\nabla_{y} S_{x} T_{x} W_{L}(x, y)\right\| \leq \kappa L^{d+1}(1+L|x-y|)^{-d-1} .
$$

This inequality implies that

$$
\begin{aligned}
& \delta \sum_{j=1}^{N} \frac{1}{N} \sup _{x \in 2 X_{j}}|S T P(x)| \\
& \leq \delta \sum_{j=1}^{N} \frac{1}{N} \sup _{x \in 2 X_{j}} \int_{\mathcal{M}}\left\|\nabla_{y} P(y)\right\|\left\|\nabla_{y} S_{x} T_{x} W_{L}(x, y)\right\| d \mu(y) \\
& \leq \kappa \delta \sum_{j=1}^{N} \frac{1}{N} \sup _{x \in 2 X_{j}} \int_{\mathcal{M}}\left\|\nabla_{y} P(y)\right\| L^{d+1}(1+L|x-y|)^{-d-1} d \mu(y) \\
& \leq \kappa \delta \int_{\mathcal{M}}\left\|\nabla_{y} P(y)\right\|\left\{\sum_{j=1}^{N} \frac{L^{d+1}}{N} \sup _{x \in 2 X_{j}}(1+L|x-y|)^{-d-1}\right\} d \mu(y) .
\end{aligned}
$$

For any fixed $y$, let now $J=\left\{j\right.$ : dist $\left.\left(2 X_{j}, y\right) \geq 2 \delta\right\}$ and $J^{\prime}$ its complement. It is easy to see that, calling $q_{j}$ the point in $2 X_{j}$ that is closest to $y$, and $p_{j}$ the point in $2 X_{j}$ that is farthest from $y$, then if $j \in J$

$$
1+\frac{L}{2}\left|p_{j}-y\right| \leq 1+\frac{L}{2}\left(\left|q_{j}-y\right|+2 \delta\right) \leq 1+L\left|q_{j}-y\right|
$$

and therefore by (2)

$$
\begin{aligned}
& \sum_{j \in J} \frac{L^{d+1}}{N} \sup _{x \in 2 X_{j}}(1+L|x-y|)^{-d-1} \\
& =\sum_{j \in J} \frac{L^{d+1}}{N}\left(1+L\left|q_{j}-y\right|\right)^{-d-1} \leq \sum_{j \in J} \frac{L^{d+1}}{N}\left(1+\frac{L}{2}\left|p_{j}-y\right|\right)^{-d-1} \\
& =\sum_{j \in J} \int_{R_{j}} L^{d+1}\left(1+\frac{L}{2}\left|p_{j}-y\right|\right)^{-d-1} d \mu(x) \\
& \leq \sum_{j \in J} \int_{R_{j}} L^{d+1}\left(1+\frac{L}{2}|x-y|\right)^{-d-1} d \mu(x) \\
& \leq \int_{\mathcal{M}} L^{d+1}\left(1+\frac{L}{2}|x-y|\right)^{-d-1} d \mu(x) \\
& \leq c_{6} L^{d+1} \int_{0}^{+\infty}\left(1+\frac{L}{2} s\right)^{-d-1} s^{d-1} d s
\end{aligned}
$$




$$
\begin{aligned}
& \leq c_{6} L^{d+1}\left(\int_{0}^{1 / L} s^{d-1} d s+\left(\frac{2}{L}\right)^{d+1} \int_{1 / L}^{+\infty} s^{-2} d s\right) \\
& \leq\left(d^{-1}+2^{d+1}\right) c_{6} L,
\end{aligned}
$$

Observe that the cardinality of $J^{\prime}$ is uniformly bounded with respect to $y$ and $N$. Indeed, the cardinality of $J^{\prime}$ is bounded above by the number of inner balls $Y_{j}$ that are contained in the ball $B(y, 4 \delta)$, and this number is bounded above by the ratio

$$
\frac{\mu(B(y, 4 \delta))}{\min _{j=1, \ldots, N} \mu\left(Y_{j}\right)} \leq \frac{c_{5}\left(8 c_{2} N^{-1 / d}\right)^{d}}{c_{4}\left(c_{1} N^{-1 / d}\right)^{d}}=\frac{c_{5}\left(8 c_{2}\right)^{d}}{c_{4} c_{1}^{d}} .
$$

Therefore, since $L \leq N^{1 / d}$,

$$
\sum_{j \in J^{\prime}} \frac{L^{d+1}}{N} \sup _{x \in 2 X_{j}}(1+L|x-y|)^{-d-1} \leq \sum_{j \in J^{\prime}} \frac{L^{d+1}}{N} \leq \frac{c_{5}\left(8 c_{2}\right)^{d}}{c_{4} c_{1}^{d}} \frac{L^{d+1}}{N} \leq \frac{8^{d} c_{5} c_{2}^{d}}{c_{4} c_{1}^{d}} L .
$$

Overall

$$
\begin{aligned}
& \left|\int_{\mathcal{M}}\|\nabla P(x)\| d \mu(x)-\sum_{j=1}^{N} \frac{1}{N}\left\|\nabla P\left(x_{j}\right)\right\|\right| \\
& \leq(2+\delta) \varepsilon+\kappa\left(\left(d^{-1}+2^{d+1}\right) c_{6}+\frac{8^{d} c_{5} c_{2}^{d}}{c_{4} c_{1}^{d}}\right) \delta L \int_{\mathcal{M}}\|\nabla P(y)\| d \mu(y) .
\end{aligned}
$$

Taking

$$
\varepsilon=\frac{\kappa\left(\left(d^{-1}+2^{d+1}\right) c_{6}+\frac{8^{d} c_{5} c_{2}^{d}}{c_{4} c_{1}^{d}}\right) \delta L}{2+\delta} \int_{\mathcal{M}}\|\nabla P(y)\| d \mu(y)
$$

we obtain

$$
\left|\int_{\mathcal{M}}\|\nabla P(x)\| d \mu(x)-\sum_{j=1}^{N} \frac{1}{N}\left\|\nabla P\left(x_{j}\right)\right\|\right| \leq C_{3} L N^{-1 / d} \int_{\mathcal{M}}\|\nabla P(y)\| d \mu(y),
$$

where

It remains to show (11). Since

$$
C_{3}=4 c_{2} \kappa\left(\left(d^{-1}+2^{d+1}\right) c_{6}+\frac{8^{d} c_{5} c_{2}^{d}}{c_{4} c_{1}^{d}}\right) .
$$

$$
\nabla_{y} S_{x} T_{x} W_{L}(x, y)=\sum_{0<\lambda_{k} \leq 2 L} \frac{1}{\lambda_{k}^{2}} h\left(\frac{\lambda_{k}}{L}\right) S T \varphi_{k}(x) \nabla \varphi_{k}(y),
$$

it is enough to estimate

$$
\sum_{0<\lambda_{k} \leq 2 L} \frac{1}{\lambda_{k}^{2}} h\left(\frac{\lambda_{k}}{L}\right) S T \varphi_{k}(x) U \varphi_{k}(y)
$$

for a generic vector field $U$ with $\|U(x)\|=1$ for all $x \in \mathcal{M}$. Since for all $u>0$ $h(u)=\sum_{j=0}^{+\infty} h\left(2^{j} u\right)-h\left(2^{j+1} u\right)$, we have

$$
\begin{aligned}
& \sum_{0<\lambda_{k}} \frac{1}{\lambda_{k}^{2}} h\left(\frac{\lambda_{k}}{L}\right) S T \varphi_{k}(x) U \varphi_{k}(y) \\
& =\sum_{j=0}^{+\infty}\left(\sum_{0<\lambda_{k}} \frac{1}{\lambda_{k}^{2}}\left(h\left(\frac{2^{j} \lambda_{k}}{L}\right)-h\left(\frac{2^{j+1} \lambda_{k}}{L}\right)\right) S T \varphi_{k}(x) U \varphi_{k}(y)\right) .
\end{aligned}
$$

Now apply Theorem 10 with $\phi_{k}=S T \varphi_{k}, \psi_{k}=U \varphi_{k}, \kappa_{1}=c_{7}(2,2), \kappa_{2}=c_{7}(1,1)$, $\kappa_{3}=c_{7}(2,1), \kappa_{4}=c_{8}(2,1), A_{1}=d+4, A_{2}=d+2, A_{3}=(d+3) / 2, S>d+3$, 
$H(u)=u^{-2}(h(2 u)-h(4 u)) \in \mathcal{C}^{S}$ and is supported in $\{1 / 4 \leq|u| \leq 1\}$. Finally replace $L$ in Theorem 10 with $2 L / 2^{j}$. Thus for $x, y \in \mathcal{M}$,

$$
\begin{aligned}
& \left|\sum_{0<\lambda_{k}} \frac{1}{\lambda_{k}^{2}}\left(h\left(\frac{2^{j} \lambda_{k}}{L}\right)-h\left(\frac{2^{j+1} \lambda_{k}}{L}\right)\right) S T \varphi_{k}(x) U \varphi_{k}(y)\right| \\
& =\left(\frac{2 L}{2^{j}}\right)^{-2}\left|\sum_{0<\lambda_{k}}\left(\frac{2 L}{2^{j} \lambda_{k}}\right)^{2}\left(h\left(\frac{2^{j} \lambda_{k}}{L}\right)-h\left(\frac{2^{j+1} \lambda_{k}}{L}\right)\right) S T \varphi_{k}(x) U \varphi_{k}(y)\right| \\
& =\left(\frac{2 L}{2^{j}}\right)^{-2}\left|\sum_{0<\lambda_{k}} H\left(\frac{2^{j} \lambda_{k}}{2 L}\right) S T \varphi_{k}(x) U \varphi_{k}(y)\right| \\
& \leq C_{9}\left(\frac{2 L}{2^{j}}\right)^{-2} \frac{\left(2 L / 2^{j}\right)^{d+3}}{\left(1+2 L 2^{-j}|x-y|\right)^{S}}=C_{9} \frac{\left(2 L 2^{-j}\right)^{d+1}}{\left(1+2 L 2^{-j}|x-y|\right)^{S}} .
\end{aligned}
$$

Adding up in $j$,

$$
\begin{aligned}
& \left|\sum_{j=0}^{+\infty}\left(\sum_{0<\lambda_{k}} \frac{1}{\lambda_{k}^{2}}\left(h\left(\frac{2^{j} \lambda_{k}}{L}\right)-h\left(\frac{2^{j+1} \lambda_{k}}{L}\right)\right) S T \varphi_{k}(x) U \varphi_{k}(y)\right)\right| \\
& \leq C_{9} \sum_{j=0}^{+\infty} \frac{\left(2 L 2^{-j}\right)^{d+1}}{\left(1+2 L 2^{-j}|x-y|\right)^{S}} .
\end{aligned}
$$

Now, if $L|x-y| \leq 1$ then

$$
\sum_{j=0}^{+\infty} \frac{\left(2 L 2^{-j}\right)^{d+1}}{\left(1+2 L 2^{-j}|x-y|\right)^{S}} \leq \sum_{j=0}^{+\infty}\left(2 L 2^{-j}\right)^{d+1} \leq 2^{d+2} L^{d+1},
$$

while if $L|x-y| \geq 1$ then

$$
\begin{aligned}
& \sum_{j=0}^{+\infty} \frac{\left(2 L 2^{-j}\right)^{d+1}}{\left(1+2 L 2^{-j}|x-y|\right)^{S}} \\
& \leq \sum_{1 \leq 2^{j} \leq L|x-y|} \frac{\left(2 L 2^{-j}\right)^{d+1}}{\left(1+2 L 2^{-j}|x-y|\right)^{S}}+\sum_{L|x-y| \leq 2^{j}} \frac{\left(2 L 2^{-j}\right)^{d+1}}{\left(1+2 L 2^{-j}|x-y|\right)^{S}} \\
& \leq \sum_{1 \leq 2^{j} \leq L|x-y|} \frac{\left(2 L 2^{-j}\right)^{d+1}}{\left(2 L 2^{-j}|x-y|\right)^{S}}+\sum_{L|x-y| \leq 2^{j}}\left(2 L 2^{-j}\right)^{d+1} \\
& \leq 2^{d+2-S} \frac{L^{d+1-S}}{|x-y|^{S}} L^{S-d-1}|x-y|^{S-d-1}+2^{d+2} L^{d+1} L^{-d-1}|x-y|^{-d-1} \\
& \leq 2^{d+3}|x-y|^{-d-1} .
\end{aligned}
$$

Overall

$$
\sum_{j=0}^{+\infty} \frac{\left(L 2^{-j}\right)^{d+1}}{\left(1+L 2^{-j}|x-y|\right)^{S}} \leq 2^{d+3} L^{d+1}(1+L|x-y|)^{-d-1} .
$$

\section{Proof of Theorem 6}

Let $\Omega$ be the open subset of the vector space $\Pi_{L}^{0} \subset L^{2}(\mathcal{M}, d \mu)$

$$
\Omega=\left\{P \in \Pi_{L}^{0}: \int_{\mathcal{M}}\|\nabla P(x)\| d \mu(x)<1\right\} .
$$


Since $\int_{\mathcal{M}}\|\nabla P(x)\| d \mu(x)$ is a norm in the finite dimensional space $\Pi_{L}^{0}$, it is equivalent to the $L^{2}$ norm in $\Pi_{L}^{0}$, and it follows that $\Omega$ is bounded in $\Pi_{L}^{0} \subset L^{2}(\mathcal{M})$, and the map from $\Pi_{L}^{0} \subset L^{2}(\mathcal{M})$ to $\mathbb{R}$ given by

$$
P \mapsto \int_{\mathcal{M}}\|\nabla P(x)\| d \mu(x)
$$

is continuous, so that $\Omega$ is open.

Lemma 14. There exists a continuous map $F: \Pi_{L}^{0} \rightarrow \mathcal{M}^{N}$ such that for every $P \in \partial \Omega$

$$
\sum_{j=1}^{N} P\left(x_{j}(P)\right)>0,
$$

where $F(P)=\left(x_{1}(P), \ldots, x_{N}(P)\right)$.

Let us first show that this lemma readily implies Theorem [6] By the Riesz representation theorem, for each point $x \in \mathcal{M}$ there exists a unique polynomial $G_{x} \in \Pi_{L}^{0}$ such that

$$
\left\langle G_{x}, P\right\rangle=P(x) \text { for all } P \in \Pi_{L}^{0} .
$$

Then a set of points $x_{1}, \ldots, x_{N} \in \mathcal{M}$ forms an $L$-design if and only if

$$
G_{x_{1}}+\cdots+G_{x_{N}}=0 .
$$

Now let $Z: \mathcal{M}^{N} \rightarrow \Pi_{L}^{0}$ be the continuous map defined by

$$
Z\left(x_{1}, \ldots, x_{N}\right)=G_{x_{1}}+\cdots+G_{x_{N}},
$$

and call $f=Z \circ F: \Pi_{L}^{0} \rightarrow \Pi_{L}^{0}$. Clearly for every $P \in \partial \Omega$ we have

$$
\langle P, f(P)\rangle=\sum_{j=1}^{N} P\left(x_{j}(P)\right)>0
$$

by the lemma, and by Theorem 2 it follows that there exists $Q \in \Omega$ such that $Z(F(Q))=0$, that is such that $G_{x_{1}(Q)}+\cdots+G_{x_{N}(Q)}=0$ which implies that $\left\{x_{1}(Q), \ldots, x_{N}(Q)\right\}$ is an $L$-design.

Proof of Lemma 14. Take a partition of $\mathcal{M}$ as in Theorem 3 with constants $c_{1}$ and $c_{2}$ and let $C_{\mathcal{M}} \geq \max \left\{1,2^{d} C_{3}\left(c_{1}, 2 c_{2}\right)^{d}, 2^{d} C_{3}\left(c_{1}, 13 c_{2}\right)^{d}\right\}$, where the constants $C_{3}(\cdot, \cdot)$ are as in Theorem 5. For each $j=1, \ldots, N$ choose an arbitrary $x_{j} \in R_{j}$.

Now fix $\varepsilon<1 / 4$ and let as before $v_{\varepsilon}:[0,+\infty) \rightarrow \mathbb{R}$ be a $\mathcal{C}^{\infty}$ function such that $v_{\varepsilon}(u)=u$ for $u \geq \varepsilon, v_{\varepsilon}(u)=\varepsilon / 2$ for $u<\varepsilon / 4$ and $v_{\varepsilon}(u) \geq u$ for all $u \geq 0$.

Take the mapping $U: \Pi_{L}^{0} \rightarrow \mathcal{X}(\mathcal{M})$ defined by

$$
U(P)(y)=\frac{\nabla P(y)}{v_{\varepsilon}(\|\nabla P(y)\|)}, \quad y \in \mathcal{M} .
$$

For each $j=1, \ldots, N$ let $y_{j}: \Pi_{L}^{0} \times[0,+\infty) \rightarrow \mathcal{M}$ be the map satisfying the differential equation

$$
\frac{d}{d t} y_{j}(P, t)=U(P)\left(y_{j}(P, t)\right)
$$

with the initial condition

$$
y_{j}(P, 0)=x_{j}
$$

for each $P \in \Pi_{L}^{0}$. Since the mapping $U(p, y)$ is Lipschitz continuous in both $P$ and $y$, each $y_{j}$ is well defined and continuous in $P$ and $t$. Now set

$$
F(P)=\left(x_{1}(P), \ldots, x_{N}(P)\right):=\left(y_{1}\left(P, 12 c_{2} N^{-1 / d}\right), \ldots, y_{N}\left(P, 12 c_{2} N^{-1 / d}\right)\right),
$$


which is continuous on $\Pi_{L}^{0}$ by definition. Let now $P \in \partial \Omega$, that is

$$
\int_{\mathcal{M}}\|\nabla P(x)\| d \mu(x)=1
$$

We have

$$
\begin{aligned}
\frac{1}{N} \sum_{j=1}^{N} P\left(x_{j}(P)\right) & =\frac{1}{N} \sum_{j=1}^{N} P\left(y_{j}\left(P, 12 c_{2} N^{-1 / d}\right)\right) \\
& =\frac{1}{N} \sum_{j=1}^{N} P\left(x_{j}\right)+\int_{0}^{12 c_{2} N^{-1 / d}} \frac{d}{d t}\left(\frac{1}{N} \sum_{j=1}^{N} P\left(y_{j}(P, t)\right)\right) d t
\end{aligned}
$$

Now,

$$
\begin{aligned}
\left|\frac{1}{N} \sum_{j=1}^{N} P\left(x_{j}\right)\right| & =\left|\sum_{j=1}^{N} \int_{R_{j}}\left(P\left(x_{j}\right)-P(x)\right) d \mu(x)\right| \leq \sum_{j=1}^{N} \int_{R_{j}}\left|P\left(x_{j}\right)-P(x)\right| d \mu(x) \\
& \leq \frac{1}{N} \sum_{j=1}^{N} \operatorname{diam}\left(R_{j}\right) \max _{z \in 2 X_{j}}\|\nabla P(z)\| \leq \frac{2 c_{2}}{N^{1+1 / d}} \sum_{j=1}^{N}\left\|\nabla P\left(z_{j}\right)\right\|,
\end{aligned}
$$

where $z_{j}$ is the point that realizes the maximum. Observe that the partition $\mathcal{R}^{\prime}=\left\{R_{1}^{\prime}, \ldots, R_{N}^{\prime}\right\}$ defined by $R_{j}^{\prime}=R_{j} \cup\left\{z_{j}\right\}$ satisfies Theorem 3 with constants $c_{1}$ and $2 c_{2}$. Therefore, by Theorem 5 applied to $P$ and the partition $\mathcal{R}^{\prime}$, since $C_{3}\left(c_{1}, 2 c_{2}\right) L N^{-1 / d} \leq\left(C_{\mathcal{M}} L^{d} N^{-1}\right)^{1 / d} / 2 \leq 1 / 2$, we have

$$
\begin{aligned}
& \left|\frac{1}{N} \sum_{j=1}^{N} P\left(x_{j}\right)\right| \leq \frac{2 c_{2}}{N^{1+1 / d}} \sum_{j=1}^{N}\left\|\nabla P\left(z_{j}\right)\right\| \\
& \leq \frac{2 c_{2}}{N^{1 / d}}\left|\sum_{j=1}^{N} \frac{1}{N}\left\|\nabla P\left(z_{j}\right)\right\|-\int_{\mathcal{M}}\|\nabla P(z)\| d \mu(z)\right|+\frac{2 c_{2}}{N^{1 / d}} \int_{\mathcal{M}}\|\nabla P(z)\| d \mu(z) \\
& \leq \frac{3 c_{2}}{N^{1 / d}} \int_{\mathcal{M}}\|\nabla P(z)\| d \mu(z)=\frac{3 c_{2}}{N^{1 / d}}
\end{aligned}
$$

for any $P \in \partial \Omega$. On the other hand, for $t \in\left[0,12 c_{2} N^{-1 / d}\right]$

$$
\begin{aligned}
\frac{d}{d t}\left(\frac{1}{N} \sum_{j=1}^{N} P\left(y_{j}(P, t)\right)\right) & =\frac{1}{N} \sum_{j=1}^{N} \frac{\left\|\nabla P\left(y_{j}(P, t)\right)\right\|^{2}}{v_{\varepsilon}\left(\left\|\nabla P\left(y_{j}(P, t)\right)\right\|\right)} \\
& \geq \frac{1}{N} \sum_{j:\left\|\nabla P\left(y_{j}(P, t)\right)\right\| \geq \varepsilon}\left\|\nabla P\left(y_{j}(P, t)\right)\right\| \\
& \geq \frac{1}{N} \sum_{j=1}^{N}\left\|\nabla P\left(y_{j}(P, t)\right)\right\|-\varepsilon .
\end{aligned}
$$

Since clearly $\left|y_{j}(P, t)-x_{j}\right| \leq t$, the partition $\mathcal{R}^{\prime \prime}=\left\{R_{1}^{\prime \prime}, \ldots, R_{N}^{\prime \prime}\right\}$ defined by $R_{j}^{\prime \prime}=R_{j} \cup\left\{y_{j}(P, t)\right\}$ satisfies Theorem 3 with the constants $c_{1}$ and $13 c_{2}$. Therefore by Theorem [5] applied to $P$ and the partition $\mathcal{R}^{\prime \prime}$, since $C_{3}\left(c_{1}, 13 c_{2}\right) L N^{-1 / d} \leq$ $\left(C_{\mathcal{M}} L^{d} N^{-1}\right)^{1 / d} / 2 \leq 1 / 2$, we have

$$
\frac{d}{d t}\left(\frac{1}{N} \sum_{j=1}^{N} P\left(y_{j}(P, t)\right)\right)
$$




$$
\begin{aligned}
& \geq \frac{1}{N} \sum_{j=1}^{N}\left\|\nabla P\left(y_{j}(P, t)\right)\right\|-\varepsilon \\
& \geq \int_{\mathcal{M}}\|\nabla P(y)\| d \mu(y)-\left|\int_{\mathcal{M}}\|\nabla P(y)\| d \mu(y)-\frac{1}{N} \sum_{j=1}^{N}\left\|\nabla P\left(y_{j}(P(t))\right)\right\|\right|-\varepsilon \\
& \geq \frac{1}{2} \int_{\mathcal{M}}\|\nabla P(y)\| d \mu(y)-\varepsilon=\frac{1}{2}-\varepsilon
\end{aligned}
$$

for each $P \in \partial \Omega$ and $t \in\left[0,12 c_{2} N^{-1 / d}\right]$. Finally,

$$
\begin{aligned}
\frac{1}{N} \sum_{j=1}^{N} P\left(x_{j}(P)\right) & =\frac{1}{N} \sum_{j=1}^{N} P\left(x_{j}\right)+\int_{0}^{12 c_{2} N^{-1 / d}} \frac{d}{d t}\left(\frac{1}{N} \sum_{j=1}^{N} P\left(y_{j}(P, t)\right)\right) d t \\
& \geq \frac{12 c_{2}}{N^{1 / d}}\left(\frac{1}{2}-\varepsilon\right)-\frac{3 c_{2}}{N^{1 / d}}=(3-12 \varepsilon) \frac{c_{2}}{N^{1 / d}}>0 .
\end{aligned}
$$

\section{REFERENCES}

[1] M. Berger, P. Gauduchon, E. Mazet, Le spectre d'une variété riemannienne, Lecture Notes in Mathematics, 194, Springer-Verlag, Berlin-New York, 1971.

[2] A. Bondarenko, D. Radchenko, M. Viazovska, Optimal asymptotic bounds for spherical designs, Ann. Math. 178, 443-452 (2013).

[3] L. Brandolini, C. Choirat, L. Colzani, G. Gigante, R. Seri, G. Travaglini, Quadrature rules and distribution of points on manifolds, Ann. Sc. Norm. Sup. Pisa Cl. Sci. (5) XIII, 889-923 (2014).

[4] L. Brandolini, W. W. L Chen, L. Colzani, G. Gigante, G. Travaglini, Discrepancy and Numerical Integration on Metric Measure Spaces, J. Geom. Anal. (2018), https://doi.org/10.1007/s12220-018-9993-6.

[5] J. S. Brauchart, J. Dick, E. B. Saff, I. H. Sloan, Y. G. Wang, R. S. Womersley, Covering of spheres by spherical caps and worst-case error for equal weight cubature in Sobolev spaces, J. Math. Anal. Appl. 431, 782-811 (2015).

[6] J. S. Brauchart, E. B. Saff, I. H. Sloan, R. S. Womersley, QMC designs: optimal order quasi Monte Carlo integration schemes on the sphere, Math. Comput. 83, 2821-2851 (2014).

[7] M. P. do Carmo, Riemannian Geometry, Translated from the second Portuguese edition by Francis Flaherty. Mathematics: Theory \& Applications, Birkhäuser Boston, Inc., Boston, MA, 1992.

[8] U. Etayo, J. Marzo, J. Ortega-Cerdà, Asymptotically optimal designs on compact algebraic manifolds, J. Monatsh. Math. 186, 235-248 (2018).

[9] F. Filbir, H. N. Mhaskar, A Quadrature Formula for Diffusion Polynomials Corresponding to a Generalized Heat Kernel, J. Fourier Anal. Appl. 16, 629-657 (2010).

[10] F. Filbir, H. N. Mhaskar, Marcinkiewicz-Zygmund measures on manifolds, J. Complexity 27, 568-596 (2011).

[11] G. Gigante, P. Leopardi, Diameter bounded equal measure partitions of Ahlfors regular metric measure spaces, Discret. Comput. Geom. 57, 419-430 (2017).

[12] P. Greiner, An asymptotic expansion for the heat equation, Arch. Rational Mech. Anal. 41, 163-218 (1971)

[13] L. Hormander, The analysis of linear partial differential operators, I II III IV, Springer Verlag, 1983-1985.

[14] Yu. A. Kordyukov, $L^{p}$ theory of elliptic differential operators on manifolds of bounded geometry, Acta Appl. Math. 23, 223-260 (1991).

[15] J. Korevaar, J. L. H. Meyers, Spherical Faraday cage for the case of equal point charges and Chebyshev-type quadrature on the sphere, Integral Transform. Spec. Funct. 1, 105117 (1993).

[16] J. M. Lee, Introduction to smooth manifolds, Second edition, Graduate Texts in Mathematics, 218, Springer, New York, 2013.

[17] M. Maggioni, H. N. Mhaskar, Diffusion polynomial frames on metric measure spaces, Appl. Comput. Harmon. Anal. 24, 329-353 (2008). 
[18] D. O'Regan, Y. J. Cho, Y.-Q. Chen, Topological Degree Theory and Applications, Ser. Math. Anal. Appl., 10, Chapman \& Hall / CRC, Boca Raton, FL, 2006.

[19] A. Sikora, Riesz transform, Gaussian bounds and the method of wave equation. Math. Z. 247, 643-662 (2004).

[20] E. M. Stein, R. Shakarchi, Complex analysis. Princeton Lectures in Analysis, 2. Princeton University Press, Princeton, NJ, 2003.

Dipartimento di Ingegneria Gestionale, dell'Informazione e della Produzione, Università degli Studi di Bergamo, Viale Marconi 5, Dalmine BG, Italy

E-mail address: biancamaria.gariboldi@unibg.it

Dipartimento di Ingegneria Gestionale, dell'Informazione e della Produzione, Università degli Studi di Bergamo, Viale Marconi 5, Dalmine BG, Italy

E-mail address: giacomo.gigante@unibg.it 